Research Article

\title{
The Gut Microbiota and Its Relevance to Peripheral Lymphocyte Subpopulations and Cytokines in Patients with Rheumatoid Arthritis
}

\author{
Yuan Li, ${ }^{1,2}$ Sheng-Xiao Zhang, ${ }^{1,2}$ Xu-Fang Yin, ${ }^{1}$ Ming-Xing Zhang, ${ }^{1}$ Jun Qiao, \\ Xiao-Hong Xin, ${ }^{3}$ Min-Jing Chang, ${ }^{1}$ Chong Gao, ${ }^{4}$ Ya-Feng Li $\left(1,{ }^{3}\right.$ and Xiao-Feng Li ${ }^{1}{ }^{1}$ \\ ${ }^{1}$ Department of Rheumatology, The Second Hospital of Shanxi Medical University, Shanxi, China \\ ${ }^{2}$ Key laboratory of Cellular Physiology at Shanxi Medical University, Ministry of Education, China \\ ${ }^{3}$ Department of Nephrology, Precision Medicine Center, The Shanxi Provincial People's Hospital, Shanxi Medical University, \\ Taiyuan, China \\ ${ }^{4}$ Department of Pathology, Brigham and Women's Hospital, Harvard Medical School, Boston, MA, USA
}

Correspondence should be addressed to Ya-Feng Li; dr.yafengli@gmail.com and Xiao-Feng Li; 1xf_9859@sxmu.edu.cn

Received 30 October 2020; Revised 24 December 2020; Accepted 28 December 2020; Published 8 January 2021

Academic Editor: Baohui Xu

Copyright ( 2021 Yuan Li et al. This is an open access article distributed under the Creative Commons Attribution License, which permits unrestricted use, distribution, and reproduction in any medium, provided the original work is properly cited.

Growing experimental and clinical evidence suggests that a chronic inflammatory response induced by gut microbiome critically contribute to the development of rheumatoid arthritis (RA). Previous studies demonstrated the disturbance of lymphocyte subpopulations in RA patients. The purpose of this study was to explore the characteristics of gut microbiome and the associations between bacterium and lymphocyte subpopulations as well as cytokines in patients with RA. Fecal samples from 205 RA patients and 199 healthy controls (HCs) were collected for bacterial DNA extraction and 16S ribosomal RNA (rRNA) gene sequencing. The levels of peripheral lymphocyte subpopulation such as $\mathrm{T}, \mathrm{B}, \mathrm{CD} 4^{+} \mathrm{T}, \mathrm{CD} 8^{+} \mathrm{T}, \mathrm{NK}, \mathrm{T}$ helper 1 (Th1), Th2, Th17, and regulatory $\mathrm{T}$ cells (Tregs) of these subjects were detected by flow cytometry combined with standard absolute counting beads. The serum levels of cytokines interleukin-2 (IL-2), IL-4, IL-6, IL-10, IL-17, tumour necrosis factor- $\alpha$ (TNF- $\alpha$ ), and interferon- $\gamma$ (INF- $\gamma$ ) were tested by flow cytometric bead array (CBA). Alpha and beta diversity of gut microbiome were explored by bioinformatics analysis. Spearman rank correlation test was used to explore the relationships between gut microbiome and lymphocyte subsets as well as serum cytokines. The diversity and relative abundance of intestinal microbiota in patients with RA were significantly different from those in HCs. Detailly, the abundant of phylum Proteobacteria in RA patients was more than that in HCs, while Firmicutes was less than in HCs. There was increased relative abundance of genus Clostridium_XlVa as well as genus Blautia, more abundance of Ruminococcus2 in patients with lower levels of T, B, CD4 ${ }^{+} \mathrm{T}$, and Tregs. In addition, the relative abundances of Pelagibacterium, Oxalobacter, ClostridiumXlVb, and ClostridiumXVIII were correlated with cytokines. Gut microbiome of RA patients was clearly different from that of HCs. Abnormal bacteria communities are associated with the altered levels of lymphocyte subpopulation and cytokines, which might be one of the pathogenesis of RA.

\section{Introduction}

Rheumatoid arthritis (RA) is a systemic autoimmune disorder characterized by a chronic immune response that leads to inflammation and destruction of synovial joints [1]. The etiological mechanisms involved are heredity, infection, and environmental trigger $[2,3]$. Accumulating evidences proposed that gut microbiota was an indispensable environmental factor in the progression of RA [4-8]. Gut microbiota, a major source of microbes, plays a key role in human body defence system $[9,10]$. Aberrant immune response was closely associated with dysbiosis of the gut microbiota $[7,8,11,12]$. 
Dysbiosis of gut microbiota triggers several types of autoimmune diseases by disturbing the balance of lymphocyte subpopulations such as $\mathrm{T}$ helper 1 (Th1), Th2, Th17, and regulatory $\mathrm{T}$ cells (Tregs) [5].

Our previous studies have demonstrated that patients with autoimmune disease have a disturbance of lymphocyte subpopulations mainly manifests as insufficient absolute counts of circulating $\mathrm{CD}^{+} \mathrm{T}$ lymphocyte subsets such as Tregs [13], which was related to disease activities and could be reversed by immunomodulatory drugs (IMiDs) such as low-dose interleukin-2 and sirolimus [14, 15]. However, there is no conclusive evidence to reveal a causal relationship between microorganism and lymphocyte subpopulations with RA.

In this study, we aimed to outline the picture of intestinal microbiota of RA and further to explore the associations between bacterium and lymphocyte subpopulations as well as cytokines.

\section{Materials and Methods}

2.1. Study Participants. A total of 404 participants were recruited in this study, comprising 205 RA patients and 199 age and sex-matched healthy controls (HCs). Patients with RA diagnosed in the Second Hospital of Shanxi Medical University were recruited between December 2018 and August 2019. All the patients met the 2010 American College of Rheumatology (ACR)/European League Against Rheumatism (EULAR) classification criteria for RA [16]. HCs were enrolled from the health examination centre of Shanxi Provincial People's Hospital. In addition, the participants who received antibiotics treatment within two months or having a known history of gastrointestinal tract disorders were excluded. Each participant provided informed consent, and the study was approved by the institutional ethics committee of the Second Affiliated Hospital of Shanxi Medical University (Ethics Number: 2019-YX-107).

2.2. Sample Collection. All fresh fecal samples collected from participants within a sterile box were transported to the laboratory immediately and then stored at $-80^{\circ} \mathrm{C}$. Among these patients, 143 of them donated their peripheral blood to test for lymphocytes, and 112 of them were agreed to test cytokine analysis. The bloods' samples were centrifuged (3000 g for $20 \mathrm{~min}$ ) within one hour of collection. After centrifugation, the plasma was used for lymphocyte subpopulation analysis and the serum for cytokine analysis.

2.3. DNA Extraction and Illumina Sequencing. Microbial genome was extracted from approximately $250 \mathrm{mg}$ fecal samples using QIAamp PowerFecal DNA Kit (Qiagen) according to the manufacturer's instructions. The quality of sample DNA was examined by using an agarose gel electrophoresis and NanoDrop One (Thermo Fisher Scientific). DNA extracts were employed for the amplification of the V3-V4 hypervariable regions of the microbial $16 \mathrm{~S}$ rRNA gene, with KAPA HiFi HotStart Ready Mix (Roche). The products were purified and recovered by FC magic beans Kit (enlighten). Qubit 4.0 (Thermo Fisher Scientific) was used to quantify
TABLE 1: Age and gender information of the enrolled participants.

\begin{tabular}{lcccc}
\hline \multirow{2}{*}{ Group } & \multirow{2}{*}{ Number } & Male & Female & Age \\
\hline HC & 199 & 81 & 118 & $50.03 \pm 11.04$ \\
RA & 205 & 75 & 130 & $52.11 \pm 12.24$ \\
$P$ & & \multicolumn{2}{c}{0.395} & 0.074 \\
\hline
\end{tabular}

the purified products. Each sample was diluted to $4 \mathrm{nM}$. Mix equal volume of each sample for pooling followed by denaturing with $\mathrm{NaOH}$, at least $5 \%$ of the Phix library, was added to balance the library polymorphism. Each sample was sequenced on Miseq PE300 (Illumina).

2.4. Bioinformatics Analysis. After filtrating and merging the raw data, UPARSE was used to select operational taxonomic units (OTUs) with a similarity cutoff of 97\% [17]. Alpha diversity was analyzed by Mothur. Beta diversity was analyzed by QIIME software package. Linear discriminant analysis (LDA) effect size (LEfSe) was analyzed to identify the community differences between groups and distinguish the biomarker species.

2.5. Assessment of Lymphocyte Subpopulations and Cytokine in Serum. A modified method of flow cytometry was used to detect the plasma levels of lymphocyte subpopulations 14 . The absolute counts and proportions of lymphocyte subpopulations including $\mathrm{T}\left(\mathrm{CD}^{+} \mathrm{CD} 45^{+}\right), \mathrm{B}\left(\mathrm{CD} 19^{+} \mathrm{CD} 45^{+}\right), \mathrm{CD} 4^{+} \mathrm{T}$ $\left(\mathrm{CD}^{+} \mathrm{CD}^{+} \mathrm{CD} 45^{+}\right), \mathrm{CD}^{+} \mathrm{T}\left(\mathrm{CD}^{+} \mathrm{CD}^{+} \mathrm{CD} 45^{+}\right)$and $\mathrm{NK}$ $\left(\mathrm{CD} 16^{+} \mathrm{CD} 56^{+} \mathrm{CD} 45^{+}\right)$, Th1 $\left(\mathrm{CD} 4^{+} \mathrm{IL}-2^{+}\right)$, Th2 $\left(\mathrm{CD} 4^{+} \mathrm{IL}-4^{+}\right)$, Th17 $\left(\mathrm{CD} 4^{+} \mathrm{IL}-17^{+}\right)$, and Tregs $\left(\mathrm{CD} 4^{+} \mathrm{CD} 25^{+} \mathrm{FoxP}^{+}\right)$. The flow cytometric bead array (CBA) was used to detect the serum levels of IL-2, IL-4, IL-6, IL-10, IL-17, TNF- $\alpha$, and INF- $\gamma$. Every procedure followed the manufacturer's instructions.

2.6. Statistical Analysis. The SPSS 22.0 statistical software was used for analysis. Alpha diversity was compared between groups using nonparametric tests (Mann-Whitney or Kruskal-Wallis); beta diversity was explored using permutational multivariate analysis of variance (PERMANOVA). Data were expressed as mean \pm standard or mean \pm standard error of mean. Group means were compared using the MannWhitney $U$ test and Student's $t$-test. Spearman rank correlation test was used for correlation analysis. $P<0.05$, the difference was statistically significant. $P<0.01$, the difference was significant.

\section{Results and Discussion}

3.1. Clinical Characteristics of Participants. The clinical information and basic characteristics of participants are showed in Table 1 . There was no statistically significant difference in age or gender between these two groups $(P>0.05)$.

3.2. GutMicrobiota ofRA Patients Differed from HCs. All samples were sequenced by high-throughput sequencing method. 5594217 high-quality reads which used to construct OTUs were obtained after filtrating and merging from these samples. Amplicons were clustered into 1051 OTUs at $97 \%$ 


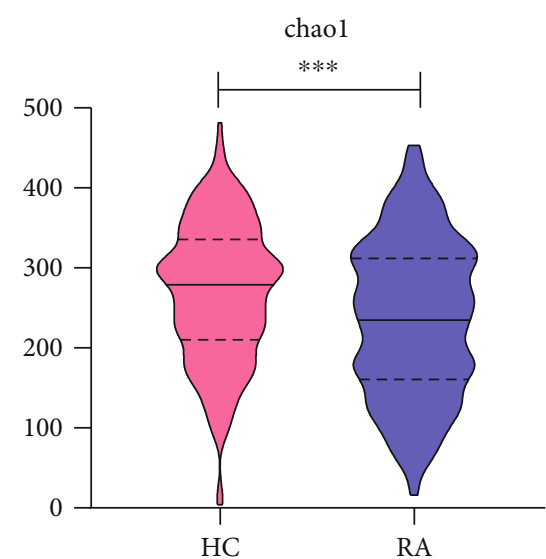

(a)

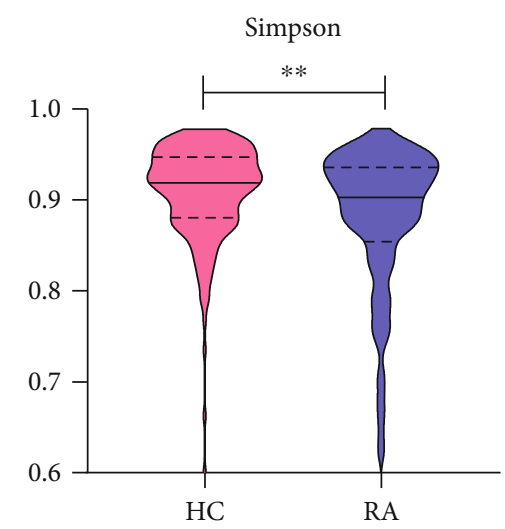

(d)

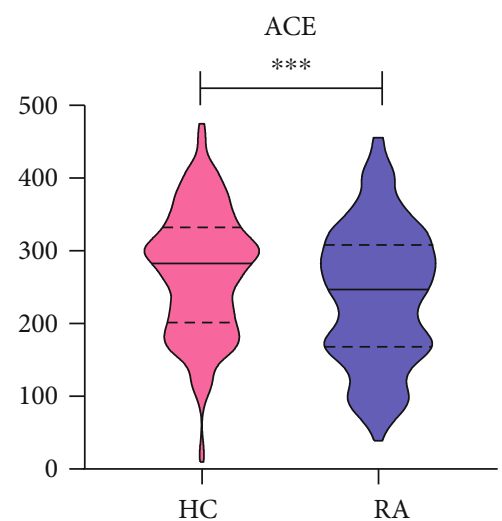

(b)

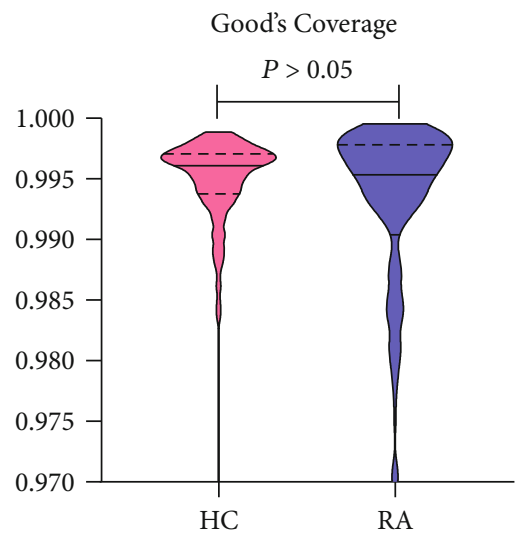

(e)

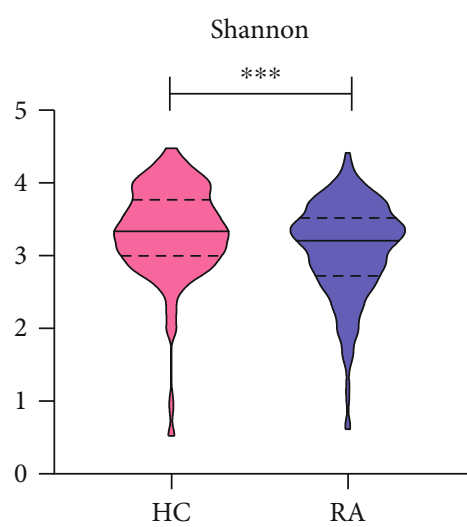

(c)

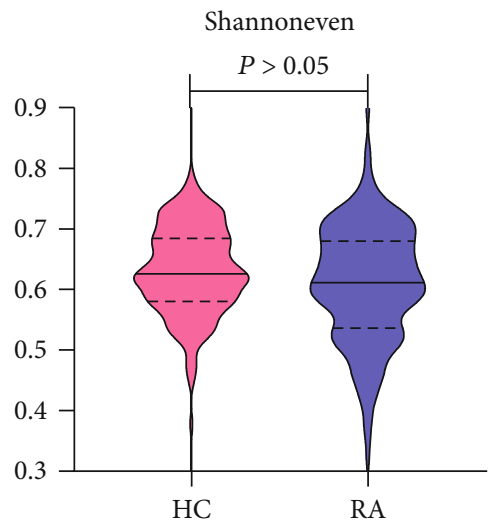

(f)

Figure 1: The $\alpha$ diversity of the gut microbiomes for the RA patients and the healthy controls has obvious differences $\left({ }^{* * *} P<0.001,{ }^{* *} P<0.01\right)$.

similarity. The rarefaction curves tended to be close to saturated platform, indicating the number of samples was ample. Similar results in the Shannon-Wiener curves and RankAbundance curves, which indicated the amount of sequencing data, were abundant enough to reflect most of the microbial species information in the sample.

To determine alpha diversity, Chaol and ACE index were used to calculate community richness. Shannon and Simpson index were able to evaluate community diversity. Gut microbiota alpha diversity assessed by the number of the observed genera significantly differed between RA and HCs. Compared with HCs, the richness and diversity of gut microbiota in RA was significantly decreased $(P<0.01$, Figure 1). No significant difference was apparent in Good's Coverage index between HCs and RA (98.99\% versus 98.59, $P>0.05$, Figure 1(e)). The higher the Good's Coverage index, the higher the probability of species being measured in the sample. There was no difference in Shannoneven index between the two groups $(P>0.05$, Figure 1(f)).

For community structure, a relative abundance at the phyla level and a relative abundance larger than $0.5 \%$ at the genus level were assessed. Compared with the HCs, the relative abundance of Proteobacteria at phyla level increased in RA, while the relative abundance of Firmicutes declined statistically $(P<0.05)$ (Figure 2(a)). At the genus level, patients with RA had an increased relative abundance of Escherichia/-
Shigella as well as Ruminococcus2 in RA $(P<0.05)$. However, the relative abundance of Lachnospiracea_incertae sedis, Prevotella, Clostridium_XlVa, Roseburia, Dialister, Unclassified_Lachnospiraceae, Blautia, Megamonas, Unclassified_Clostridiales, Gemmiger, Parasutterella, Acetivibrio, Coprococcus, and Anaerostipes were declined in RA $(P<0.05)$ (Figure 2(b)). Noticeably, there were also significant differences in the relative abundance of Clostridium XVIII, Clostridium_XlVb, Lactobacillus, and so on between the two groups $(P<0.05)$ (Figure 3$)$.

The Bray-Curtis distance was used to calculate beta diversity. PERMANOVA also demonstrated that the microbiota composition significantly differed between the two groups $(P<0.01)$.

3.3. Alteration in Gut Microbiota between RA and HCs. Based on the results of species-abundance comparison between two groups of gut microbiota, the community differences between groups were analyzed from the level of phylum to genus classification by using linear discriminant analysis effect size (LEfSe) [18] (Figure 4). Our results suggested that the relative abundance of the phylum Proteobacteria, along with its three families, Hyphomicrobiaceae, Enterobacteriaceae, and Halomonadaceae, were increased in RA patients compared with HCs $(P<0.05)$. In HCs, phylum Firmicutes had the largest LDA score. 


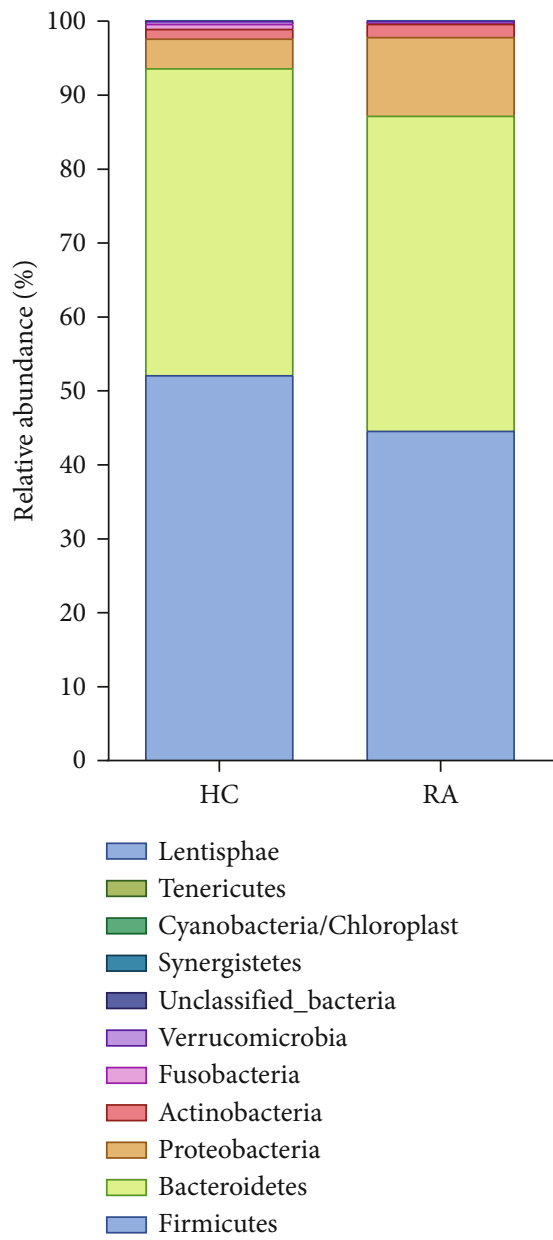

(a)

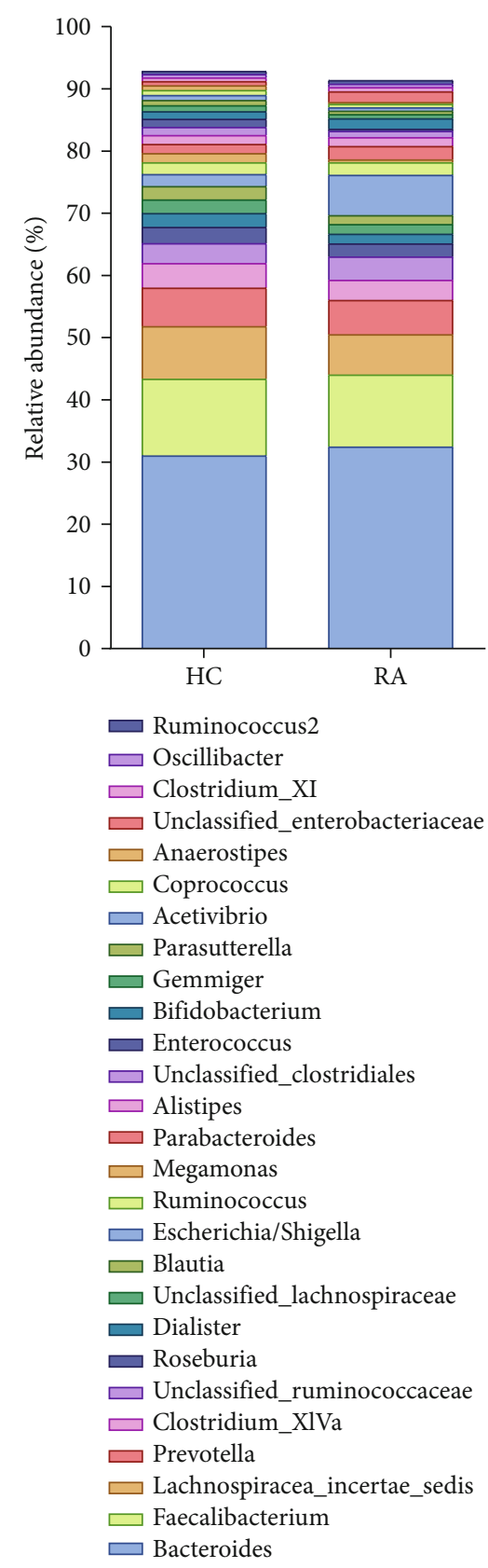

(b)

Figure 2: Microbiota composition of healthy controls and RA patients at the phylum (a) and genus (b) levels.

3.4. The Relevance between Different Gut Microbial Genera and Peripheral Lymphocyte Subpopulations. Considering the correlation between intestinal microorganisms and peripheral blood (PB) lymphocyte subpopulations, taxa that showed significant difference in relative abundance were selected to evaluate the relevance between gut microbiota composition and lymphocyte subpopulations.

Spearman correlation coefficients were computed between each bacterial taxa and each of the plasma levels of $\mathrm{T}, \mathrm{B}$, $\mathrm{CD} 4^{+} \mathrm{T}, \mathrm{CD} 8^{+} \mathrm{T}$, and $\mathrm{NK}$ cells. The relative abundance of Blautia, Ruminococcus2, and Odoribacter showed significant negative correlations with these markers $(P<0.05)$. Detailly, Blautia was related to the absolute numbers of $\mathrm{B}$ and $\mathrm{CD} 4^{+} \mathrm{T}$ cells $(P<0.05)$. Ruminococcus 2 was related to the absolute numbers of T, B, and $\mathrm{CD} 4^{+} \mathrm{T}$ cells $(P<0.05)$. Besides, the relative abundance of Pelagibacterium was positively correlated with the absolute numbers of B cells $(P<0.05)$ (Figure 5).

As for $\mathrm{CD}^{+} \mathrm{T}$ cell subsets such as Th1, Th2, Th17, and Tregs, the relative abundances of Anaerostipes, Blautia, Odoribacter, and Ruminococcus 2 were significantly and negatively correlated with these markers $(P<0.05)$. Additionally, the relative abundance of Cloacibacillus was positively correlated with the absolute numbers of Th2 $(P<0.05)$ and Th17 $(P<0.01)$, and the relative abundance of Streptophyta showed a positive correlation with the absolute numbers of Th17 $(P<0.05)$ (Figure 6). 


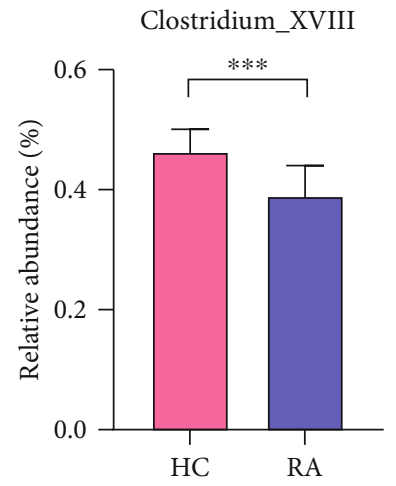

(a)

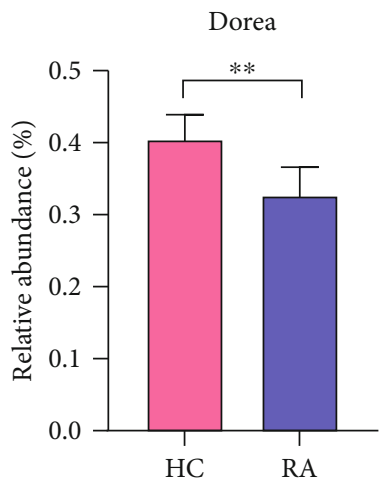

(b)

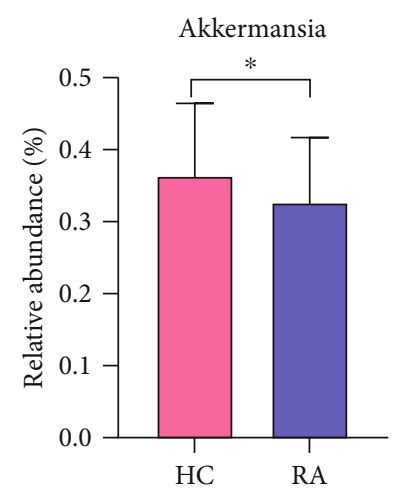

(c)

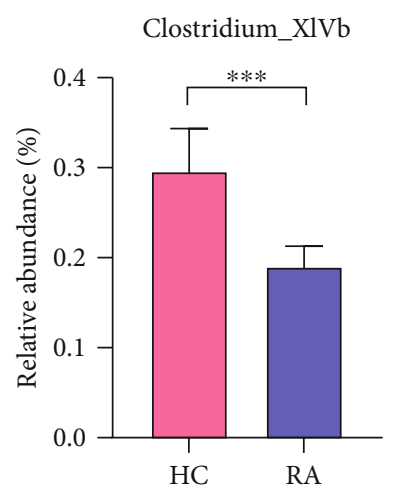

(d)

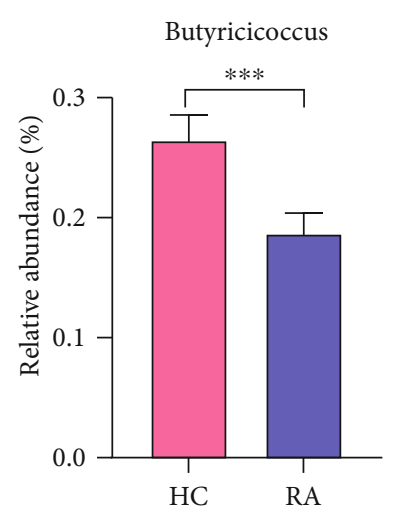

(e)

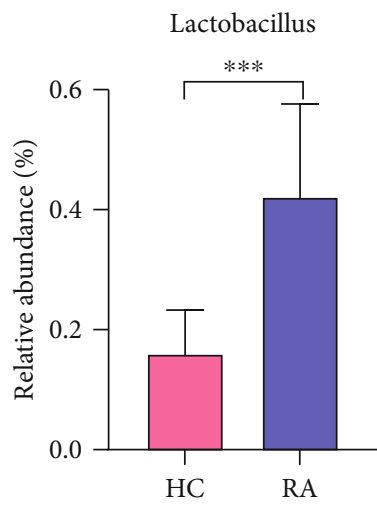

(f)

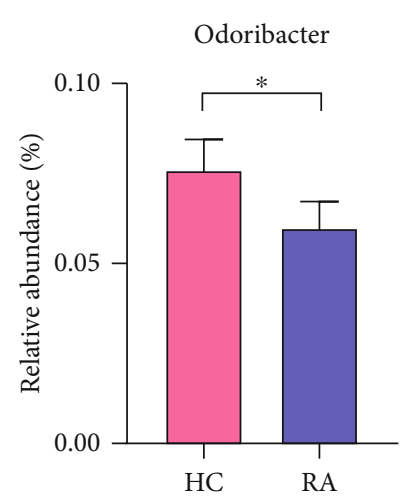

(g)

FIgURE 3: At the generic level, the relative abundances of some gut microbiome in healthy controls and RA patients were compared. Data were expressed as mean \pm standard error of mean $\left({ }^{* * *} P<0.001,{ }^{* *} P<0.01,{ }^{*} P<0.05\right)$.

3.5. The Relevance between Different Gut Microbial Genera and Cytokines. Based on the results that analyzed by LEfSe, taxa that showed significant difference in relative abundance were selected to evaluate the relevance between gut microbiota composition and cytokines. Spearman correlation coefficients were computed between each bacterial taxa and each of the serum levels of IL-2, IL-4, IL-6, IL-10, IL-17, TNF- $\alpha$, and INF- $\gamma$. As showed in Figure 7, the relative abundance of Pelagibacterium had a significant positive correlation with these cytokines $(P<0.05)$. On the contrary, the relative abundance of Oxalobacter was negatively correlated with these cytokines $(P<0.05)$. There was less abundance of Unclassified-Bacteroidetes in patients with lower levels of IL-2 $(P<0.05)$, IL-10 $(P<0.05)$, IL-17 $(P<0.01)$, INF- $\gamma$ $(P<0.05)$, and TNF- $\alpha(P<0.05)$. The relative abundances of ClostridiumXlVb and ClostridiumXVIII are positively correlated with IL-2 and IL-10, respectively $(P<0.05)$. These findings indicated different gut microbiota closely associated with serum cytokines in RA.

\section{Discussion}

The microbiome components are an attractive source of antigens capable of inducing RA and have been the most investigated as potential causal agents [19]. Consistent with previous studies [20-22], our findings identified gut micro- biome alteration in individuals with RA by both alpha diversity and beta diversity.

Specific gut microbiota played an important role in the pathogenesis of RA. In our research, phylum Proteobacteria, the largest phylum of bacteria, including many pathogenic bacteria, such as Enterobacter and Salmonella, were more abundant in RA patients. These pathogens may invade the intestinal mucosa to increase its permeability, the proliferation of inflammatory cells, and secretion of inflammatory mediators of them, leading to immune inflammatory response. At the genus level, patients with RA displayed a mean 3-fold overabundance of Lactobacillu, which characterized a potentially harmful microorganisms and participated in the development of inflammatory arthritis [23]. Further, Lactobacillu was successfully used to induce arthritis model because Lactobacillu might result in an imbalance of Th17/Tregs homeostasis by mediating TLR2-TLR4 signaling [24]. In addition, some studies have also shown that Lactobacillus casei (L. casei) supplementation has an active improvement for patients with RA $[25,26]$, suggesting different species of Lactobacillus have different effects on arthritis, and further detailed research is needed.

We found insufficient abundance of Clostridium_XlVa, Clostridium_XlVb, and Clostridium_XVIII in patients with RA. Interestingly, intestinal Clostridia species was reported to alleviate inflammatory response by produce IL-10 through specific capsule component polysaccharide A (PSA) and 


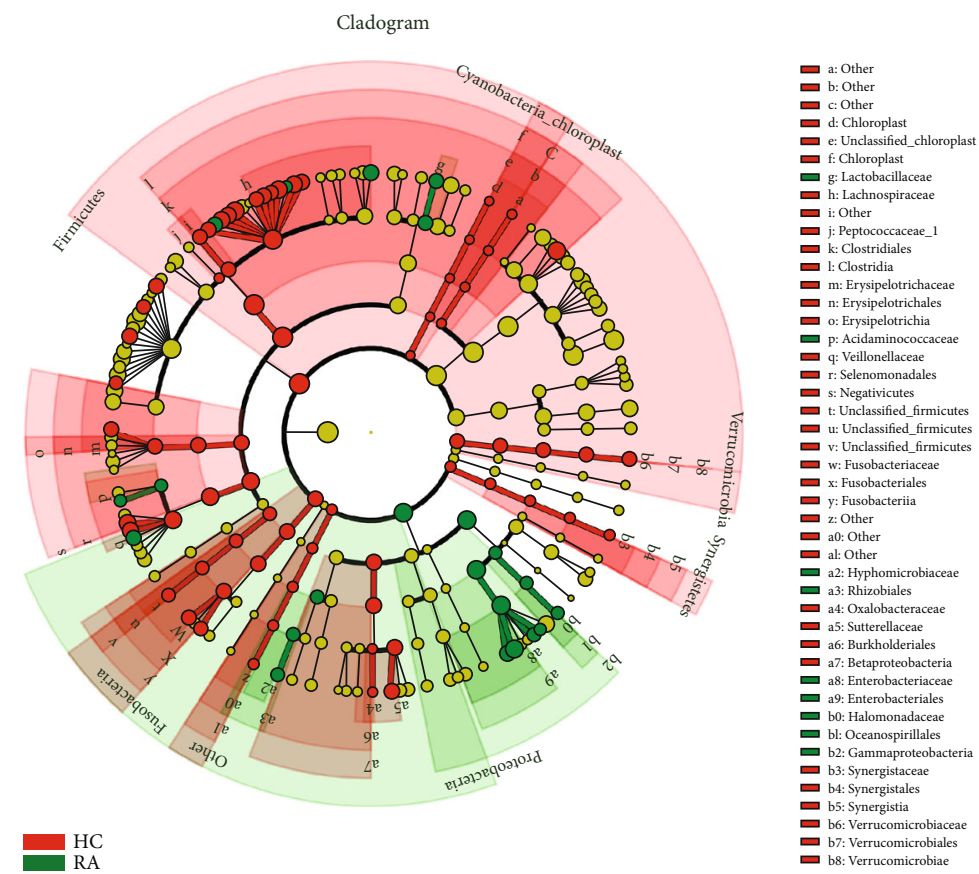

(a)

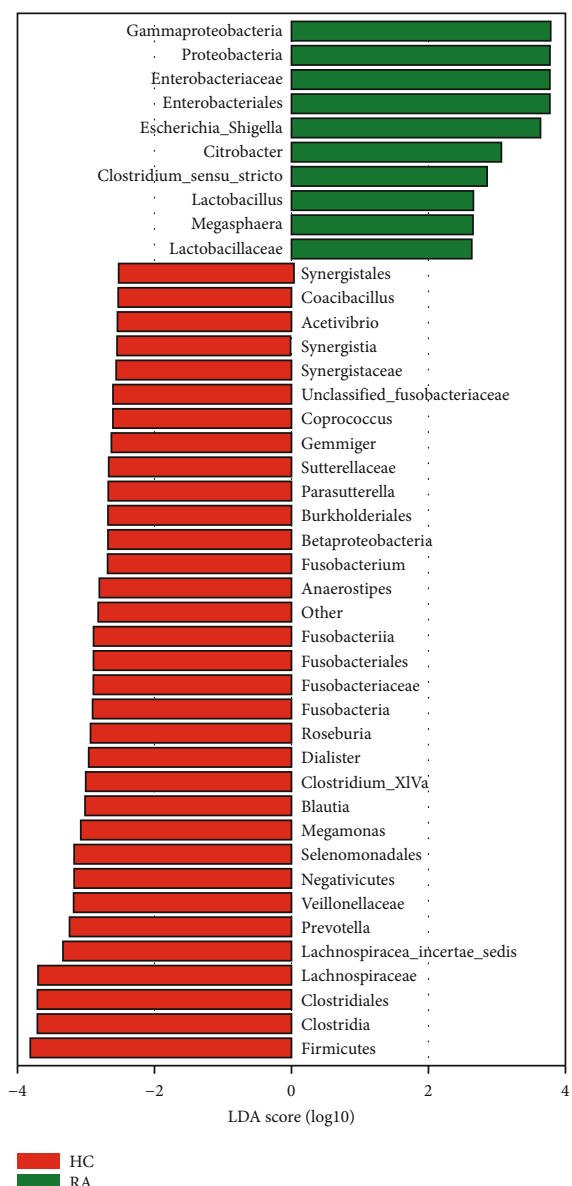

(b)

FIGURE 4: (a, b) LefSe analysis was performed to identify differentially abundant taxa, which are highlighted on the phylogenetic tree in cladogram format (a) and for which the LDA scores are shown (b). 


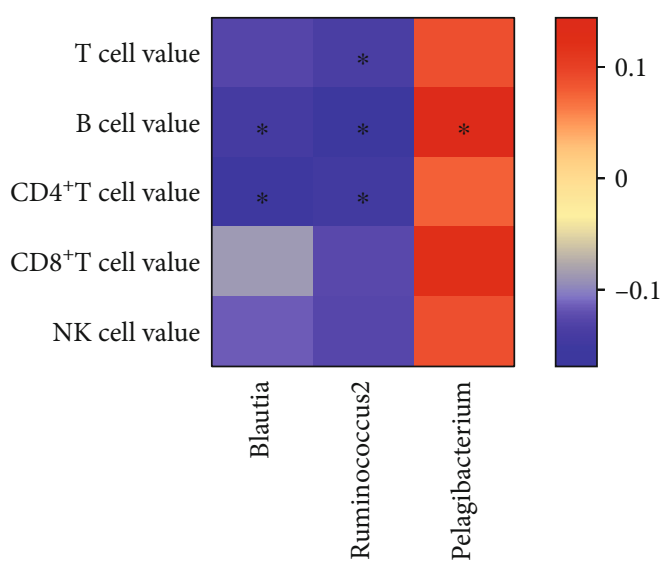

Figure 5: A heat map shows the correlation between different gut microbial species and lymphocyte subpopulations. Colors indicate the Spearman rank correlation $\left({ }^{*} P<0.05\right)$.

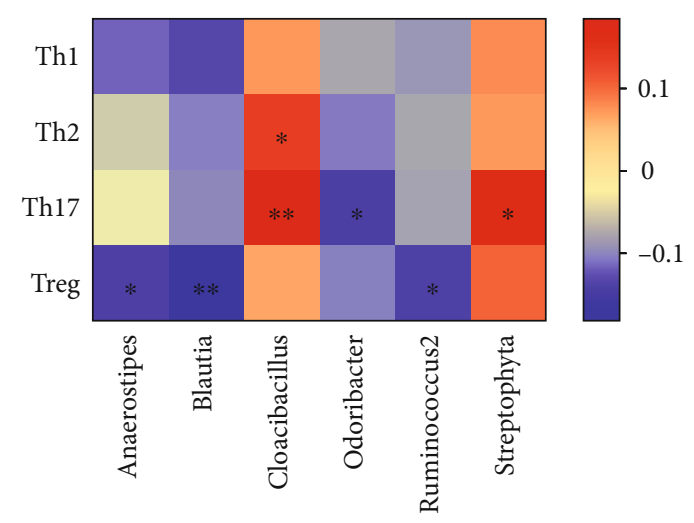

Figure 6: A heat map shows the correlation between different gut microbial species and $\mathrm{CD} 4^{+} \mathrm{T}$ lymphocyte subpopulations. Colors indicate the Spearman rank correlation $\left({ }^{* *} P<0.01,{ }^{*} P<0.05\right)$.

induce Treg proliferation [27]. Clostridium_XlVa has antiinflammatory properties by enhancing transforming growth factor-beta (TGF- $\beta$ ) expression and inducting or accumulating Tregs [27-29]. Research shows that Treg can maintain tolerance to autoantigens and eliminate autoimmunity and play an important role in maintaining immune tolerance to dietary antigens and intestinal flora [30]. The decrease in the number and function of Treg cells leads to the deficiency of immune tolerance and abnormal immune response to autoantigens, which are involved in the occurrence and development of RA [31]. As Tregs play a significant role in anti-inflammation and maintaining immune system homeostasis [32], insufficiency of these floras might attenuate Tregs promoting the deficiency of immune tolerance.

To data, the existing research findings about Prevotella are controversial. Vaahtovuo et al. [11] found that the relative abundance of Prevotella in the intestine of RA patients decreased, which was consistent with our findings. But Prevotella copri increased among new-onset untreated RA patients $[8,33]$. Also, Rodrigues et al. [34] found an increase in relative expression units (REU) of Prevotella species in stool samples from Brazilian RA patients. In fact, for the existing disputes, further research and exploration are needed. It is

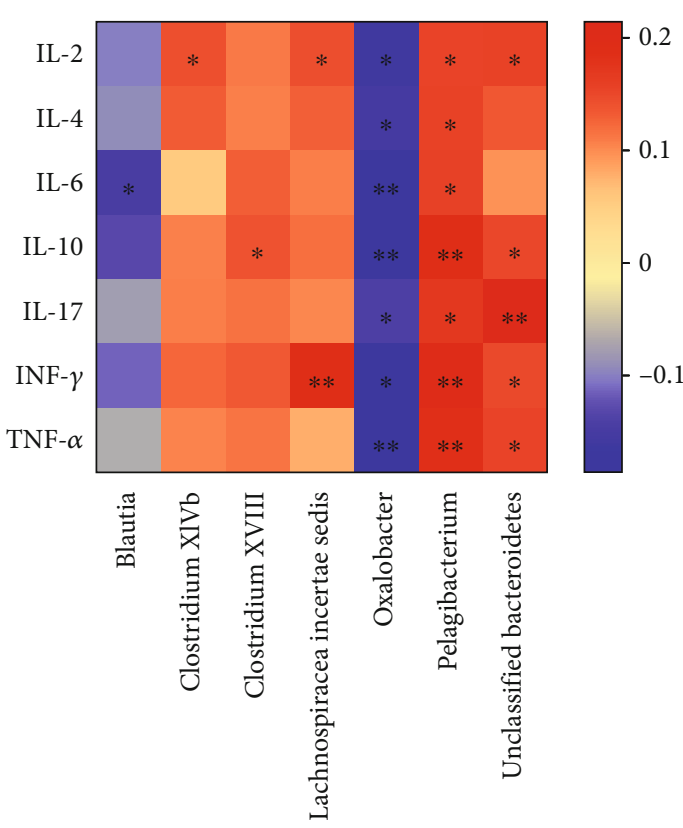

FIgURE 7: A heat map shows the correlation between different gut microbial species and cytokines. Colors indicate the Spearman rank correlation (** unadjusted $P<0.01,{ }^{*} P<0.05$ ).

confirmed that Escherichia play a role in the pathogenesis of arthritis in germ-free arthritis-prone rats [35], which is consistent with our results that showed an increase in the relative abundance of Escherichia in patients with RA.

Immunopathogenesis of RA is mainly manifested in immune system dysregulation which is characterized by the presence of autoantibodies and autoreactive $\mathrm{T}$ cells. It was found that the function of circulating Treg cells in plasma and synovium is impaired [36], and Th17 cell abundance increased $[37,38]$. The imbalance of Th17/Treg is the main factor for the abnormality of autoreactive T cells. Presumably, the imbalance of intestinal flora in susceptible hosts may destroy the balance of Th17/Treg [39], which leads to the destruction of immune tolerance, following by a systemic immune disequilibrium. This immunologic derangement favors proinflammatory responses resulting in joints and other tissue damage. Our results showed that the relative abundance of Ruminococcus2 increased in RA patients and negatively correlated with the absolute number of $\mathrm{T}, \mathrm{B}$, $\mathrm{CD}^{+} \mathrm{T}$, and Tregs, while the relative abundance of Blautia was decreased and negatively correlated with $\mathrm{B}$ and $\mathrm{CD} 4^{+} \mathrm{T}$ cell. The correlation analysis between intestinal microorganisms and lymphocyte suggests that alteration of gut microbiota might play a role in the pathogenesis of RA by modulating the immune systems of human.

Cytokines played an important role in the occurrence of inflammation and the pathogenesis of autoimmune diseases $[40,41]$. TNF- $\alpha$ plays a fundamental role through activation of cytokine and chemokine expression, expression of endothelial-cell adhesion molecules, protection of synovial fibroblasts, promotion of angiogenesis, suppression of regulatory T cells, and induction of pain $[2,42,43]$. Pelagibacterium, Oxalobacter, and Blautia were related to TNF- $\alpha$. IL-6 and IL-17 are closely related to the bone erosion by 
enhancing the differentiation and activation of osteoclast [44]. In our study, the relative abundance of Pelagibacterium was increased while Oxalobacter and Blautia decreased. Just as we expected, Pelagibacterium showed a significant positive correlation with these cytokines; Oxalobacter and Blautia were negatively correlated with these cytokines, suggesting intestinal microflora might participate in pathogenic progress by altering levels of cytokines.

\section{Conclusions}

Richness and diversity of intestinal flora in RA patients were impaired, which might participate in the pathogenesis of RA by modulating the immune systems with lymphocyte subpopulations and cytokines. The discovery of the associated intestinal microbiota of RA may provide a new idea for RA treatment.

\section{Data Availability}

The SRA data used to support the findings of this study are available from the corresponding author.

\section{Disclosure}

The abstract of this manuscript was presented as a poster at Annual meeting of Chinese Physiological Society.

\section{Conflicts of Interest}

The authors declare that there is no conflict of interest regarding the publication of this paper.

\section{Acknowledgments}

We thank all the fecal donors who participated in our study. This project was supported by the National Science Foundation of China (81871295, 82001740), the Open Fund from the Key Laboratory of Cellular Physiology (Shanxi Medical University) (KLCP2019), and the Innovation Plan for Postgraduate Education in Shanxi Province (2020BY078).

\section{References}

[1] J. A. Hill, D. A. Bell, W. Brintnell et al., "Arthritis induced by posttranslationally modified (citrullinated) fibrinogen in DR4-IE transgenic mice," The Journal of Experimental Medicine, vol. 205, no. 4, pp. 967-979, 2008.

[2] I. B. McInnes and G. Schett, "The pathogenesis of rheumatoid arthritis," The New England Journal of Medicine, vol. 365, no. 23, pp. 2205-2219, 2011.

[3] D. L. Scott, F. Wolfe, and T. W. Huizinga, "Rheumatoid arthritis," Lancet, vol. 376, no. 9746, pp. 1094-1108, 2010.

[4] J. Chen, K. Wright, J. M. Davis et al., "An expansion of rare lineage intestinal microbes characterizes rheumatoid arthritis," Genome Medicine, vol. 8, no. 1, article 43, 2016.

[5] N. Lee and W. U. Kim, "Microbiota in T-cell homeostasis and inflammatory diseases," Experimental \& Molecular Medicine, vol. 49, no. 5, p. e340, 2017.
[6] X. Liu, Q. Zou, B. Zeng, Y. Fang, and H. Wei, "Analysis of fecal Lactobacillus community structure in patients with early rheumatoid arthritis," Current Microbiology, vol. 67, no. 2, pp. 170176, 2013.

[7] J. U. Scher, A. Sczesnak, R. S. Longman et al., "Expansion of intestinal Prevotella copri correlates with enhanced susceptibility to arthritis," eLife, vol. 2, article e01202, 2013.

[8] X. Zhang, D. Zhang, H. Jia et al., "The oral and gut microbiomes are perturbed in rheumatoid arthritis and partly normalized after treatment," Nature Medicine, vol. 21, no. 8, pp. 895-905, 2015.

[9] Y. Belkaid and T. W. Hand, "Role of the microbiota in immunity and inflammation," Cell, vol. 157, no. 1, pp. 121-141, 2014.

[10] L. V. Hooper and A. J. Macpherson, "Immune adaptations that maintain homeostasis with the intestinal microbiota," Nature Reviews. Immunology, vol. 10, no. 3, pp. 159-169, 2010.

[11] J. Vaahtovuo, E. Munukka, M. Korkeamäki, R. Luukkainen, and P. Toivanen, "Fecal microbiota in early rheumatoid arthritis," The Journal of Rheumatology, vol. 35, no. 8, pp. 15001505, 2008.

[12] V. Taneja, "Arthritis susceptibility and the gut microbiome," FEBS Letters, vol. 588, no. 22, pp. 4244-4249, 2014.

[13] S. X. Zhang, J. Wang, J. W. Chen et al., "The level of peripheral regulatory $\mathrm{T}$ cells is linked to changes in gut commensal microflora in patients with systemic lupus erythematosus," Annals of the Rheumatic Diseases, 2019.

[14] M. Miao, Z. Hao, Y. Guo et al., "Short-term and low-dose IL-2 therapy restores the Th17/Treg balance in the peripheral blood of patients with primary Sjogren's syndrome," Annals of the Rheumatic Diseases, vol. 77, no. 12, pp. 1838-1840, 2018.

[15] S. X. Zhang, J. Wang, H. H. Sun et al., "Circulating regulatory $\mathrm{T}$ cells were absolutely decreased in dermatomyositis/polymyositis patients and restored by low-dose IL-2," Annals of the Rheumatic Diseases, 2019.

[16] D. Aletaha, T. Neogi, A. J. Silman et al., "2010 rheumatoid arthritis classification criteria: an American College of Rheumatology/European League Against Rheumatism collaborative initiative," Annals of the Rheumatic Diseases, vol. 69, no. 9, pp. 1580-1588, 2010.

[17] B. M. Goebel and E. Stackebrandt, "Cultural and phylogenetic analysis of mixed microbial populations found in natural and commercial bioleaching environments," Applied and Environmental Microbiology, vol. 60, no. 5, pp. 1614-1621, 1994.

[18] N. Segata, J. Izard, L. Waldron et al., "Metagenomic biomarker discovery and explanation," Genome Biology, vol. 12, no. 6, p. R60, 2011.

[19] L. R. Espinoza and I. Garcia-Valladares, "Of bugs and joints: the relationship between infection and joints," Reumatología Clínica (English Edition), vol. 9, no. 4, pp. 229-238, 2013.

[20] M. Breban, J. Tap, A. Leboime et al., "Faecal microbiota study reveals specific dysbiosis in spondyloarthritis," Annals of the Rheumatic Diseases, vol. 76, no. 9, pp. 1614-1622, 2017.

[21] H. I. Chiang, J. R. Li, C. C. Liu et al., “An association of gut microbiota with different phenotypes in Chinese patients with rheumatoid arthritis," Journal of Clinical Medicine, vol. 8, no. 11, 2019.

[22] Y. Maeda, A. Kumanogoh, and K. Takeda, "Altered composition of gut microbiota in rheumatoid arthritis patients," Nihon Rinshō Men'eki Gakkai Kaishi, vol. 39, no. 1, pp. 59-63, 2016. 
[23] J. U. Scher and S. B. Abramson, "The microbiome and rheumatoid arthritis," Nature Reviews Rheumatology, vol. 7, no. 10, pp. 569-578, 2011.

[24] S. Abdollahi-Roodsaz, L. A. Joosten, M. I. Koenders et al., "Stimulation of TLR2 and TLR4 differentially skews the balance of T cells in a mouse model of arthritis," The Journal of Clinical Investigation, vol. 118, no. 1, pp. 205-216, 2008.

[25] B. Alipour, A. Homayouni-Rad, E. Vaghef-Mehrabany et al., "Effects of Lactobacillus casei supplementation on disease activity and inflammatory cytokines in rheumatoid arthritis patients: a randomized double-blind clinical trial," International Journal of Rheumatic Diseases, vol. 17, no. 5, pp. 519527, 2014.

[26] E. Vaghef-Mehrabany, B. Alipour, A. Homayouni-Rad, S. K. Sharif, M. Asghari-Jafarabadi, and S. Zavvari, "Probiotic supplementation improves inflammatory status in patients with rheumatoid arthritis," Nutrition, vol. 30, no. 4, pp. 430-435, 2014.

[27] K. Atarashi, T. Tanoue, T. Shima et al., "Induction of colonic regulatory T cells by indigenous Clostridium species," Science, vol. 331, no. 6015, pp. 337-341, 2011.

[28] Y. Sun, Q. Chen, P. Lin et al., "Characteristics of gut microbiota in patients with rheumatoid arthritis in Shanghai, China," Frontiers in Cellular and Infection Microbiology, vol. 9, p. 369, 2019.

[29] T. Chiba and H. Seno, "Indigenous clostridium species regulate systemic immune responses by induction of colonic regulatory T cells," Gastroenterology, vol. 141, no. 3, pp. 11141116, 2011.

[30] K. Honda and D. R. Littman, "The microbiota in adaptive immune homeostasis and disease," Nature, vol. 535, no. 7610, pp. 75-84, 2016.

[31] B. Prakken, W. Ellen, and F. van Wijk, "Editorial: quality or quantity? Unraveling the role of Treg cells in rheumatoid arthritis," Arthritis and Rheumatism, vol. 65, no. 3, pp. 552554, 2013.

[32] J. H. Esensten, Y. D. Muller, J. A. Bluestone, and Q. Tang, "Regulatory T-cell therapy for autoimmune and autoinflammatory diseases: the next frontier," The Journal of Allergy and Clinical Immunology, vol. 142, no. 6, pp. 1710-1718, 2018.

[33] N. J. Bernard, "Rheumatoid arthritis: Prevotella copri associated with new-onset untreated RA," Nature Reviews Rheumatology, vol. 10, no. 1, p. 2, 2014.

[34] G. S. Rodrigues, L. C. Cayres, F. P. Gonçalves et al., "Detection of increased relative expression units of Bacteroides and Prevotella, and decreased Clostridium leptum in stool samples from Brazilian rheumatoid arthritis patients: a pilot study," Microorganisms, vol. 7, no. 10, 2019.

[35] O. Kohashi, Y. Kohashi, T. Takahashi, A. Ozawa, and N. Shigematsu, "Suppressive effect of Escherichia coli on adjuvant-induced arthritis in germ-free rats," Arthritis and Rheumatism, vol. 29, no. 4, pp. 547-553, 1986.

[36] A. Zanin-Zhorov, Y. Ding, S. Kumari et al., "Protein kinase Ctheta mediates negative feedback on regulatory $\mathrm{T}$ cell function," Science, vol. 328, no. 5976, pp. 372-376, 2010.

[37] A. Hot and P. Miossec, "Effects of interleukin (IL)-17A and IL$17 \mathrm{~F}$ in human rheumatoid arthritis synoviocytes," Annals of the Rheumatic Diseases, vol. 70, no. 5, pp. 727-732, 2011.

[38] E. M. Colin, P. S. Asmawidjaja, J. P. van Hamburg et al., "1,25dihydroxyvitamin D3 modulates Th17 polarization and interleukin-22 expression by memory $\mathrm{T}$ cells from patients with early rheumatoid arthritis," Arthritis and Rheumatism, vol. 62, no. 1, pp. 132-142, 2010.

[39] B. Chen, L. Sun, and X. Zhang, "Integration of microbiome and epigenome to decipher the pathogenesis of autoimmune diseases," Journal of Autoimmunity, vol. 83, pp. 31-42, 2017.

[40] L. T. Osnes, B. Nakken, E. Bodolay, and P. Szodoray, "Assessment of intracellular cytokines and regulatory cells in patients with autoimmune diseases and primary immunodeficiencies novel tool for diagnostics and patient follow-up," Autoimmunity Reviews, vol. 12, no. 10, pp. 967-971, 2013.

[41] S. Li, D. Yang, T. Peng, Y. Wu, Z. Tian, and B. Ni, "Innate lymphoid cell-derived cytokines in autoimmune diseases," Journal of Autoimmunity, vol. 83, pp. 62-72, 2017.

[42] M. Feldmann, F. M. Brennan, and R. N. Maini, "Rheumatoid arthritis," Cell, vol. 85, no. 3, pp. 307-310, 1996.

[43] A. Hess, R. Axmann, J. Rech et al., "Blockade of TNF-alpha rapidly inhibits pain responses in the central nervous system," Proceedings of the National Academy of Sciences of the United States of America, vol. 108, no. 9, pp. 3731-3736, 2011.

[44] G. Schett and S. L. Teitelbaum, "Osteoclasts and arthritis," Journal of Bone and Mineral Research, vol. 24, no. 7, pp. 1142-1146, 2009. 